\title{
Cell Derived Hierarchical Assembly of a Novel Phosphophoryn-Based Biomaterial
}

\author{
Jinhua Li ${ }^{a}$ Dana Olton ${ }^{a, b, c, d}$ Donghyun Lee ${ }^{a, b, c, d}$ Prashant N. Kumta ${ }^{a, b, c, d}$ \\ Charles Sfeir ${ }^{a, b}$ \\ ${ }^{a}$ Center for Craniofacial Regeneration, School of Dental Medicine, and Departments of ${ }^{b}$ Bioengineering, ${ }^{\mathrm{c}} \mathrm{Chemical}$ \\ Engineering and ${ }^{\mathrm{d}}$ Mechanical Engineering and Materials Science, University of Pittsburgh, Pittsburgh, Pa., USA
}

\author{
Key Words \\ Phosphophoryn · SIBLING · Self-assembly • \\ Phosphoproteins
}

\begin{abstract}
Phosphophoryn (PP) is an acidic phosphoprotein belonging to the small integrin-binding ligand $\mathrm{N}$-linked glycoprotein (SIBLING) protein family. PP is highly phosphorylated with approximately 200 phosphates per molecule and has a high affinity for calcium. The aim of this manuscript is to demonstrate that PP has the ability to self-assemble when it is overexpressed in a mammalian cell in the presence of calcium. Our data show that when PP is overexpressed using an adenovirus, the self-assembly occurs in the endoplasmic reticulum (ER) which contains high calcium concentration. We hypothesize that the physicochemical properties of the highly phosphorylated state and acidic nature of PP are playing an important role in its assembly in the ER. It appears that when a critical concentration of PP is reached, the assembly is then favored and facilitated. This self-assembly could be due to several factors. (1) The ER provides an ideal environment for this phenomenon to occur, since the ER environment usually promotes aggregation [Stevens and Argon: Semin Cell Dev Biol 1999;10:443-454]. (2) In addition to PP's physicochemical properties, the unfolded protein response could also be playing a role in this self-assembly [Schroder and Kaufman: Mutat Res 2005;569:29-63]. Unfolded protein response could be activated by a broad spectrum of insults that result in protein misfolding and ultimately blocking of the protein synthesis
\end{abstract}

progression to the Golgi apparatus resulting in an accumulation of the protein in the ER. In summary, our data show that PP has the ability to self-assemble in a hierarchical manner.

Copyright $\odot 2008$ S. Karger AG, Basel

\section{Introduction}

Bone and dentin synthesize and assemble an extracellular matrix (ECM) into which mineral may be deposited. Type I collagen is the major protein constituent of the bone and dentin ECM, comprising approximately $90 \%$ of the total protein matrix [Hunter et al., 1996; Shi et al., 1996; Anselme 2000]. The remaining 5-10\% is composed of noncollagenous proteins which are believed to play a role in the formation of mineralized tissues [Veis, 1993;

\begin{tabular}{ll}
\hline Abbreviations used in this paper \\
\hline Ad-PP & adenovirus expressing PP \\
DGP & dentin glycoprotein \\
DSP & dentin sialoprotein \\
DSPP & dentin phosphosialoprotein \\
ECM & extracellular matrix \\
ER & endoplasmic reticulum \\
hMSCs & human mesenchymal cells \\
PCR & polymerase chain reaction \\
PP & phosphophoryn \\
SIBLING & small integrin-binding ligand N-linked glycoprotein \\
TEM & transmission electron microscopy
\end{tabular}

\section{KARGER}

() 2008 S. Karger AG, Basel

Fax +4161306 1234

E-Mail karger@karger.ch

www.karger.com
Accessible online at: www.karger.com/cto
Dr. Charles Sfeir

Center for Craniofacial Regeneration, University of Pittsburgh 693A Salk Hall, 3501 Terrace Street

Pittsburgh, PA 15261-1964 (USA)

Tel. +1 412648 1949, Fax +1 412624 6685, E-Mail csfeir@pitt.edu 
MacDougall et al., 1997; Anselme 2000; Narayanan et al., 2001]. Some ECM proteins belong to the small integrinbinding ligand N-linked glycoprotein (SIBLING) family consisting of bone sialoprotein, osteopontin, dentin matrix protein 1 and dentin sialophosphoprotein (DSPP) [Fisher et al., 2001]. These SIBLING proteins are acidic in nature and bind $\mathrm{Ca}^{2+}$ and hydroxyapatite [Veis and Perry, 1967; Young et al., 1992; MacDougall et al., 1997; Veis et al., 1998; He et al., 2003]. One of these proteins, phosphophoryn (PP), is a cleavage product of DSPP [MacDougall et al., 1997] and possesses one of the highest $\mathrm{Ca}^{2+}$ binding affinities within this group of proteins. This is due to its extreme acidity [Veis and Perry, 1967] arising from its high aspartic acid and phosphoserine serine content. Of the serine residues, $85-90 \%$ are phosphorylated [Richardson et al., 1978; Linde et al., 1980]. As we mentioned above, $\mathrm{PP}$ is a cleavage product of DSPP, the transcript which is thought to be translated as a fusion protein consisting of dentin sialoprotein (DSP)/dentin glycoprotein (DGP) and PP [Yamakoshi et al., 2006]. In our efforts to elucidate the function of PP in biomineralization, we designed a set of experiments to overexpress $\mathrm{PP}$ in mammalian cells and assess its role in matrix mineralization. In this paper, we describe the consequences of overexpressing PP in mammalian cells. We hypothesize that the overexpression of PP will shed light on PP's role in biomineralization by its interaction with other proteins or its self-assembly.

\section{Materials and Methods}

\section{Recombinant Adenovirus Construction and Virus}

\section{Purification}

To clone the mouse PP gene into the pShooter (pCMV/myc/ER; Invitrogen) vector, polymerase chain reaction (PCR) of exon 5 using the DSPP genomic DNA as a template was performed. The amplified 2-kb fragment of exon 5 was digested using SalI and $X b a I$ restriction enzymes and was subcloned into the pShooter vector. The pShooter/ER vector is designed to add a signal peptide at the $\mathrm{N}$ terminus of the coding sequence to direct protein expression to the endoplasmic reticulum (ER). The pShooter/ER-PP clone was then used as a template to amplify by PCR the PP sequence containing the signal peptide. The following primers were used: 5'-GCTTACTGGCACGTGGTACCTAATACGAC-3' (forward) and 5'-GCATCTCGAGTTAAAGCACCCGCCATTCAAATC-3' (reverse). The obtained PP fragment from the PCR reaction was then inserted into the pShuttle-CMV (AdEasy Adenoviral Vector System Cat. No. 240007) following KpnI and XhoI restriction enzyme digestion. A recombinant adenovirus was produced by a double-recombination event of cotransformed adenoviral backbone plasmid pAdEasy-1 (AdEasy Adenoviral Vector System Cat. No. 240005) and a linearized pShuttle-CMV-PP with pmeI. BJ5183 electroporation-competent cells were employed for cotransformation. Positive clones were selected and confirmed by
DNA Miniprep and pacI digestion. The resulting adenoviral plasmid DNA containing the PP fragment (Ad-PP) was then digested by pacI. Twenty micrograms of pacI-linearized Ad-PP was transfected into Ad-293 cell (Cat. No. 240085; Stratagene) using Lipofectamine 2000 (Invitrogen) as a transfection agent in a $10-\mathrm{cm}$ dish. Transfected Ad-293 cells were incubated for 8 days and their cytopathic effect on the 293 cells was observed. This was seen by the changes in cell morphology, the cells rounded up and detached from the dish. The virus was harvested, amplified and titered according to the manufacturer's recommendation (Stratagene).

\section{Cell Culture}

U2-OS human osteosarcoma cell line (HTB 96; ATCC) was maintained in McCoy's 5A medium (Hyclone) supplemented with $10 \%$ fetal calf serum. Human mesenchymal stem cells (hMSCs) from the Tulane University Vector Core Facility were cultured in $\alpha$-MEM supplemented with $16 \%$ fetal calf serum, $2 \mu \mathrm{M}$ L-glutamine (Gibco) and $100 \mathrm{U} / \mathrm{ml}$ penicillin/streptomycin. All cell lines were maintained in a humidified atmosphere of $5 \% \mathrm{CO}_{2}$ at $37^{\circ} \mathrm{C}$.

\section{Transmission Electron Microscopy}

Cells grown in 35-mm dishes were fixed for $60 \mathrm{~min}$ at room temperature and then overnight at $4^{\circ} \mathrm{C}$ in $2.5 \%$ glutaraldehyde. Following fixation, the tissue was washed 3 times in $\mathrm{PBS}$ and then postfixed in aqueous $1 \% \mathrm{OsO}_{4}, 1 \% \mathrm{~K}_{3} \mathrm{Fe}(\mathrm{CN})_{6}$ for $1 \mathrm{~h}$. The tissue was then dehydrated through a graded series of $30-100 \%$ ethanol, then infiltrated and embedded in Polybed 812 epoxy resin (Polysciences). Ultrathin $(60 \mathrm{~nm})$ sections were collected on copper grids, stained with $2 \%$ uranyl acetate in $50 \%$ methanol for $10 \mathrm{~min}$, followed by $1 \%$ lead citrate for $7 \mathrm{~min}$. Sections were studied using a JEOL JEM 1210 transmission electron microscope at $80 \mathrm{kV}$.

\section{Results}

\section{Adenovirus Expression of $P P$}

The PP sequence that consists mainly of the DSS motif (fig. 1) was expressed in mammalian cells by using an adenovirus. PP expression from the adenovirus was confirmed by performing Western blots, dot blot analyses using an anti-PP antibody and SDS gel electrophoresis using Stains-All stain that stains acidic phosphoproteins.

\section{Overexpression of PP in hMSCs or U2-OS Cells}

hMSCs or U2-OS cells were infected at an MOI of 200 with Ad-PP. The infected cells were allowed to grow in tissue culture for a week. The overexpression of PP in the infected cells was observed at about $48 \mathrm{~h}$ after infection for hMSCs and at about $16 \mathrm{~h}$ for U2-OS cells in the form of an intracellular structure that is visible by low-magnification $(\times 10)$ bright field microscopy as shown in figure 2 . This structure appears to grow axially and transversally intracellularly to form hierarchical needle-like structures. The longitudinal growth is more pronounced compared to the transverse growth, resulting in a long needle-like struc- 
ESGSRGDASYTSDESSDDDNDSDSHAGEDDSSDDSSGDGDSDSNGDGDSESEDKDESDSSDH DNSSDSESKSDSSDSSDDSSDSSDSSDSSDSSDSSDSSDSSDSSDSSDSNSSSDSSDSSGSSDSSDS SDTCDSSDSSDSSDSSDSSDSSDSSDSSDSSDSSDSSDSSSSSDSSDSSSCSDSSDSSDSSDSSDSS DSSDSSSSDSSSSSNSSDSSDSSDSSSSSDSSDSSDSSDSSDSSGSSDSSDSSASSDSSSSSDSSDSS SSSDSSDSSDSSDSSDSSESSDSSNSSDSSDSSDSSDSSDSSDSSDSSDSSDSSNSSDSSDSSDSSDS SDSSNSSDSSDSSDSSDSSDSSDSSDSSDSSDSSDSSDSSDSSDSSDSSDSSDSSDSSDSSDSSDSS 1 DSSDSSNSSDSSDSDSKDSSSDSSDGDSKSGNGNSDSNSDSNSDSDSDSEGSDSNHSTSDD.

Fig. 1. The mouse PP sequence cloned into the adenovirus and expressed.

Fig. 2. Bright field micrograph showing an hMSC infected with the Ad-PP virus. A needle-like structure is observed intracellularly in the longitudinal axis of the cell.
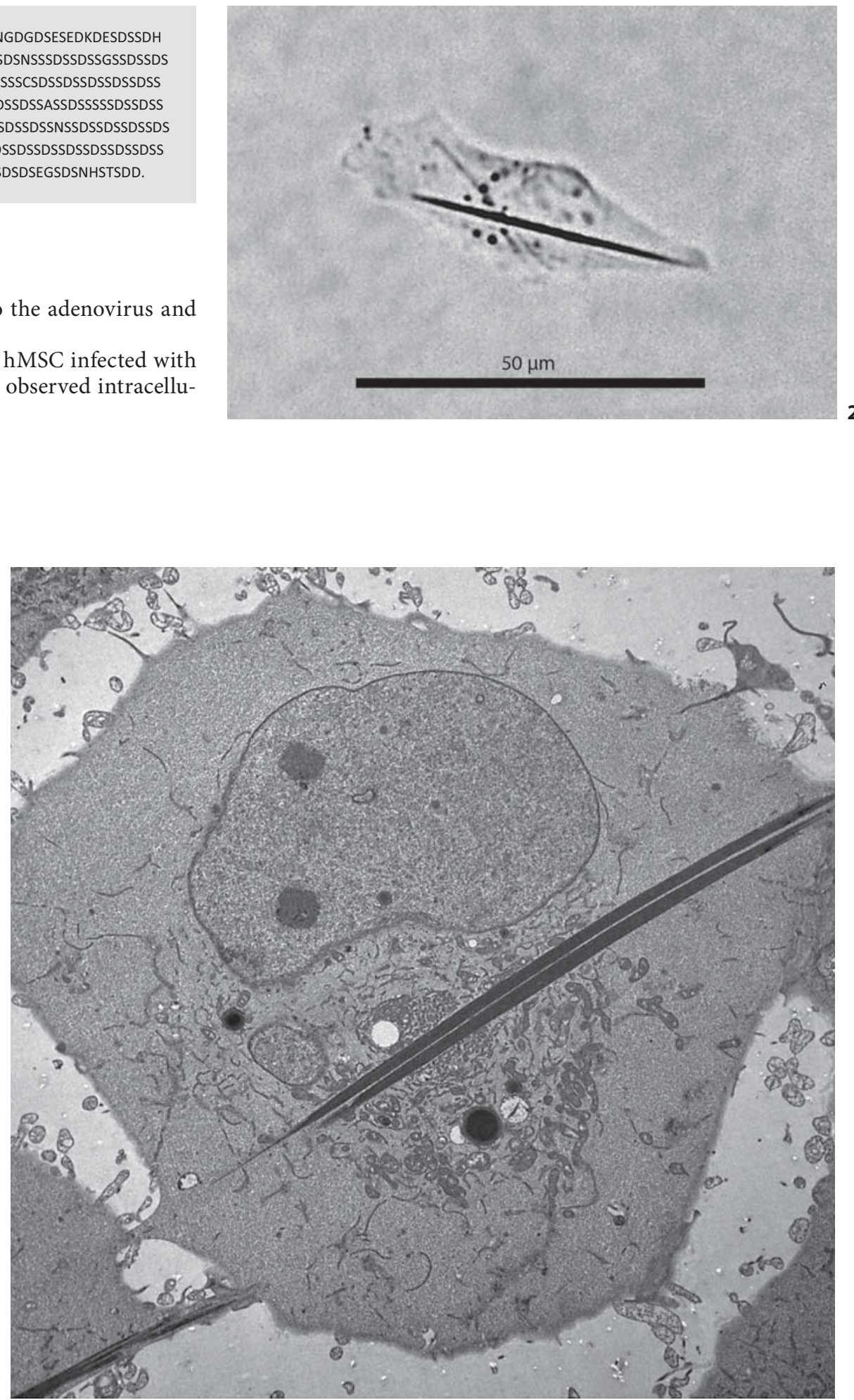

49-04B 24h-04.tif

Print Mag:4350x@7.0 in $14: 2411 / 21 / 06$
2 microns

$\mathrm{HV}=80 \mathrm{kV}$

Direct Mag: 4000x

Center for Biologic Imaging
Fig. 3. Transmission electron micrograph
showing a fibril-like structure in the ER. 
ture. The percentage of cells that assemble PP in a needlelike structure varies by cell types. U2-OS showed $100 \%$ of cells with needle-like structures, whereas hMSCs show approximately $80 \%$ of cells with needle-like structures. The growing needles stretch the cell membrane to its physical limit and lead to cell death at approximately day 6.

\section{Transmission Electron Microscopy Analysis}

Cells were fixed and processed for transmission electron microscopy analysis following their infection with Ad-PP. The needle-like structures are formed in the ER of the cell (fig. 3). In some cells, multiple structures can be seen which are at different assembly stages. It appears that the shape of the needle-like structures is directed by the ER membranes and, as observed in some micrographs, the ER membrane could be seen advancing ahead of the needles.

\section{Discussion}

The observation that when we overexpress PP in the cells it can form a needle-like structure is both exciting and unexpected. These results demonstrate that under certain conditions, PP has the ability to self-assemble into needles. We believe that these needles have the potential to serve as new protein-based biomaterials. The fact that this assembly occurs intracellularly in ER was quite unexpected, considering that PP is an extracellular protein. This, however, raises very interesting questions of the causes and mechanisms of this assembly. Is it due to physicochemical properties of the high phosphorylation state of PP that triggered the assembly? Or is it the overwhelming synthesis of PP that activated an unfolded protein response, blocked the synthesis of $\mathrm{PP}$ from progressing to the Golgi, thus allowing PP to accumulate in the ER, and provided the appropriate conditions for assembly? It appears that for the assembly to occur, a certain critical concentration of $\mathrm{PP}$ is required. This was deduced from the fact that there is a lag of $48 \mathrm{~h}$ prior to the commencement of the assembly and our ability to purify PP during this time frame. One must ask the question as to why DSP/ DGP and PP are synthesized as a fusion protein that is later cleaved. One possibility is that one of DSP/DGP's roles is to prevent PP from self-assembly during the synthesis process. There are many biological and assembly questions that need to be answered and these are the foci of our future research. These include assessment of the mechanical properties of these needle-like structures, the mechanism(s) of assembly and the question whether these needles are formed of pure PP or other proteins participating in the self-assembly. In summary, we demonstrate that a novel material could be generated by overexpressing PP in mammalian cells. These data show that PP has the ability to self-assemble and this model system could be used to study ER protein synthesis mechanisms.

\section{Acknowledgements}

C.S. and P.N.K. acknowledge the support of NIH5R01DE016123; C.S. acknowledges the assistance of NIH5K02DE016900. P.N.K. acknowledges the Edward R. Weidlein Chair Professorship funds.

\section{References}

Anselme, K. (2000) Osteoblast adhesion on biomaterials. Biomaterials 21: 667-681.

Fisher, L.W., D.A. Torchia, B. Fohr, M.F. Young, N.S. Fedarko (2001) Flexible structures of SIBLING proteins, bone sialoprotein, and osteopontin. Biochem Biophys Res Commun 280: 460-465.

-He, G., T. Dahl, A. Veis, A. George (2003) Dentin matrix protein 1 initiates hydroxyapatite formation in vitro. Connect Tissue Res 44(suppl 1): 240-245.

Hunter, G.K., P.V. Hauschka, A.R. Poole, L.C. Rosenberg, H.A. Goldberg (1996) Nucleation and inhibition of hydroxyapatite formation by mineralized tissue proteins. Biochem J 317: 59-64.

Linde, A., M. Bhown, W.T. Butler (1980) Noncollagenous proteins of dentin: a re-examination of proteins from rat incisor dentin utilizing techniques to avoid artifacts. J Biol Chem 255: 5931-5942.
MacDougall, M., D. Simmons, X. Luan, J. Nydegger, J. Feng, T.T. Gu (1997) Dentin phosphoprotein and dentin sialoprotein are cleavage products expressed from a single transcript coded by a gene on human chromosome 4: dentin phosphoprotein DNA sequence determination. J Biol Chem 272: 835-842.

Narayanan, K., R. Srinivas, A. Ramachandran, J. Hao, B. Quinn, A. George (2001) Differentiation of embryonic mesenchymal cells to odontoblast-like cells by overexpression of dentin matrix protein 1. Proc Natl Acad Sci USA 98: 4516-4521.

Richardson, W.S., E.C. Munksgaard, W.T. Butler (1978) Rat incisor phosphoprotein: the nature of the phosphate and quantitation of the phosphoserine. J Biol Chem 253: 80428046.

Shi, S., M. Kirk, A.J. Kahn (1996) The role of type I collagen in the regulation of the osteoblast phenotype. J Bone Miner Res 11: 1139-1145.
Veis, A. (1993) Mineral-matrix interactions in bone and dentin. J Bone Miner Res 8(suppl 2): S493-497.

-Veis, A., A. Perry (1967) The phosphoprotein of the dentin matrix. Biochemistry 6: 24092416.

-Veis, A., K. Wei, C. Sfeir, A. George, J. Malone (1998) Properties of the (DSS)n triplet repeat domain of rat dentin phosphophoryn. Eur J Oral Sci 106(suppl 1): 234-238.

Yamakoshi, Y., J.C. Hu, T. Iwata, K. Kobayashi, M. Fukae, J.P. Simmer (2006) Dentin sialophosphoprotein is processed by MMP-2 and MMP-20 in vitro and in vivo. J Biol Chem 281: 38235-38243.

Young, M.F., J.M. Kerr, K. Ibaraki, A.M. Heegaard, P.G. Robey (1992) Structure, expression, and regulation of the major noncollagenous matrix proteins of bone. Clin Orthop Relat Res 281: 275-294. 